\title{
Moderation of Fatigue and Stress in the Carry-over of Self-Regulation and Self-Efficacy for Exercise to Self-Regulation and Self-Efficacy for Managed Eating
}

\author{
James J. Annesi \\ YMCA of Metropolitan Atlanta, Atlanta, Georgia, USA. \\ Email: JamesA@ymcaatlanta.org \\ Received July $14^{\text {th }}, 2011$; revised August $28^{\text {th }}, 2011$; accepted October $1^{\text {st }}, 2011$.
}

\begin{abstract}
Behavioral treatments for morbid obesity have not been effective, possibly because of a poor understanding of the relations of psychosocial factors and exercise and eating behaviors. Recent research suggests that exercise program-induced improvements in self-efficacy and self-regulatory skills use may carry-over to self-efficacy and self-regulation for controlled eating. However, for individuals with morbid obesity, fatigue and anxiety may moderate these relationships. The purpose of this research was to evaluate this moderation. Adults with Grade 3 obesity $\left(M_{\mathrm{BMI}}=46.0 \mathrm{~kg} / \mathrm{m}^{2}\right)$ participated in 26 weeks of cognitive-behaviorally supported exercise paired with 12 weeks of either nutrition education $(n=95)$ or a cognitive-behavioral nutrition component $(n=109)$. There were significant improvements in self-regulation and self-efficacy for exercise, and self-regulation and self-efficacy for controlled eating, which did not differ by treatment condition. Bivariate relationships between changes in self-regulation for exercise and self-regulation for controlled eating $(\beta=.63)$, and changes in exercise self-efficacy and self-efficacy for controlled eating $(\beta=.51)$, were strong. Moderation of these relationships by fatigue and anxiety was either significant or marginally significant ( $p s<.01$ and $p$ s $<.08$, respectively). Both changes in self-regulation for controlled eating and self-efficacy for controlled eating significantly contributed to the explained variance in BMI change $\left(R^{2}=.30\right)$. Implications of the findings for behavioral weight-loss treatment for those with morbid obesity were discussed.
\end{abstract}

Keywords: Self-Regulation, Self-Efficacy, Exercise, Obesity, Nutrition

\section{Introduction}

Approximately 6\% (13.5 million) of adults in the U.S. have Grade 3 , or morbid, obesity (body mass index [BMI] $\geq 40$ $\mathrm{kg} / \mathrm{m}^{2}$ ) compared to the $34 \%$ that reach the threshold for Grade 1 obesity (BMI $\geq 30 \mathrm{~kg} / \mathrm{m}^{2}$ ) or the $68 \%$ who are overweight (BMI of 25 to $29.9 \mathrm{~kg} / \mathrm{m}^{2}$ ) or above (Flegal, Carroll, Ogden, \& Curtin, 2010). Health risks such as hypertension, hyperlipidemia, and Type 2 diabetes increase exponentially as degree of excess weight increases (Mokdad et al., 2003). Psychosocial factors such as anxiety, fatigue, self-efficacy, and coping are predictably worse in individuals with obesity than those of a healthy weight (Greenberg, Perna, Kaplan, \& Sullivan, 2005). Moreover, it is likely that the effect of these constructs on eating and exercise behaviors is distinct in persons with morbid obesity (Davies, 2007), and thus may require specific study (Annesi \& Whitaker, 2010). The lack of understanding of potentially modifiable psychological predictors of weight-management behaviors may contribute to the overwhelming failure of behavioral weight-loss treatments (Mann et al., 2007) that increasingly yield to invasive and expensive medical procedures such as gastric bypass and gastric banding surgery (Bult, Van Dalen, \& Muller, 2008) (that possess health risks of their own; Maggard et al., 2005).

Because of it being the strongest predictor of sustained weight loss (Fogelholm \& Kukkomen-Harjula, 2000; Svetkey et al., 2008), exploration of the role of exercise, beyond simple caloric expenditure (that is typically minimal in deconditioned and obese individuals; American College of Sports Medicine, 2009), has been strongly encouraged (Mann et al., 2007). Following from a social cognitive and self-efficacy framework
(Bandura, 1986, 1997), it has been suggested that exercise may have important linkages to individuals' control over excess eating through psychosocial pathways such as the effect of physical activity on perceived control, feelings of competence, and mood (Baker \& Brownell, 2000). Examples of recently identified relationships having treatment implications include associations between self-regulation and self-efficacy improvements and improvements in exercise and eating behaviors; and carry-over effects of self-regulation for exercise to self-regulation for eating, and exercise-related self-efficacy to self-efficacy for controlling eating (Annesi \& Marti, 2011; Hankonen, Absetz, Haukkala, \& Uutela, 2009; Oaten \& Cheng, 2006; Teixeira et al., 2010).

However, because completion of physical activity may be exceedingly difficult, unpleasant, and, ultimately, a different experience for individuals with morbid obesity, it is not known if these relationships generalize to them. Self-efficacy and persistence with self-regulatory skills may also be affected in individuals with morbid obesity, who are typically viewed critically by society (Puhl \& Brownell, 2002). Also, although exercise program-induced improvements in mood (e.g., anxiety, fatigue, depression) have been shown to improve emotional and otherwise uncontrolled eating (Annesi \& Marti, 2011), mood may play a quite different role in persons with morbid obesity. For example, the heightened stress and fatigue associated with this disorder may adversely affect carry-over from exercise-related self-regulation and self-efficacy to self-regulation and selfefficacy related to managed eating. Previous research suggests that fatigue and stress affects coping skills and feelings of ability to effectively deal with barriers (Hagger, Wood, Stiff, \& Chatzisarantis, 2009; Tice \& Bratslavsky, 2000). 
Thus, various research questions were tested here to aid in the ultimate development of an original, theoretically driven behavioral treatment for morbid obesity that may have better sustained effects than those currently available. In the present experimental design, cognitive-behaviorally supported exercise was incorporated because it provided impetus for analyses of the transfer of self-regulation and self-efficacy for exercise to self-regulation and self-efficacy for controlled eating and weight loss. Based on recent suggestions (Glasgow, 2008; Glasgow \& Emmons, 2007), a naturalistic environment was chosen for the investigation to maximize external validity and rapid generalizability of findings to practice settings.

Several hypotheses were proposed:

1) It was expected that the addition of a cognitive-behaviorally based nutrition component to the exercise support would be associated with greater improvements in self-regulatory skill usage and self-efficacy for controlled eating when contrasted with the addition of a more typical approach to nutrition that emphasized education in healthy eating.

2) It was also expected that, consistent with findings of individuals with varied of degrees of overweight (Annesi \& Marti, 2011), the relationships between self-regulation for exercise and self-regulation for eating, and self-efficacy for exercise and self-efficacy for controlled eating would be strong.

3) It was thought that both fatigue and stress (tension) would significantly moderate the aforementioned relationships in the present sample.

Hopefully, a better understanding of interrelations between social cognitive theory variables related to exercise, eating, and weight control in adults with Grade 3 obesity will prove useful to both theory and the development of more successful behavioral treatments.

\section{Method}

\section{Participants}

Men and women responded to advertisements soliciting volunteers for research on exercise and nutrition instruction for weight loss. Inclusion criteria were a minimum age of 21 years, a BMI of 40 to $60 \mathrm{~kg} / \mathrm{m}^{2}$ (Grade 3, or morbid, obesity), and no regular exercise within the previous year. Present or soonplanned pregnancy and use of medications for weight loss or a psychological condition were reasons for exclusion. A written statement of adequate health to participate was required from a physician. Appropriate institutional review board approval and written informed consent from all participants was obtained.

There was no significant difference in percentage of women (overall $84 \%$ ), age (overall $M=41.6$ years, $S D=10.4$ ), BMI (overall $M=46.0 \mathrm{~kg} / \mathrm{m}^{2}, S D=4.9$ ), and racial make-up (overall $44 \%$ White, $53 \%$ Black, and $3 \%$ of other racial groups) between participants randomly assigned to cognitive-behavioral exercise support plus nutrition education (Nutrition Education group; $n=95$ ) and cognitive-behavioral exercise support plus cognitive-behavioral methods applied to nutritional change (Behavioral Nutrition group; $n=109$ ). Most participants were middle-class. There was minimal attrition from initial acceptance into the study to actual treatment participation (4\%) that did not differ by group.

\section{Measures}

Self-efficacy for exercise (perceived ability to overcome barriers to completing exercise) was measured by the 5-item Exercise Self-Efficacy Scale (Marcus, Selby, Niaura, \& Rossi,
1992). It requires responses that begin with the stem, "I am confident I can participate in regular exercise when" e.g., "I have more enjoyable things to do", ranging from 1 (Not at all confident) to 7 (Very confident). Internal consistencies ranged from .76 to .82 , and test-retest reliability over 2 weeks was .90 (McAuley \& Mihalko, 1998).

Self-efficacy for controlled eating (perceived ability to overcome barriers to managing eating) was measured by the 20-item Weight Efficacy Lifestyle Questionnaire (Clark, Abrams, Niaura, Eaton, \& Rossi, 1991). Responses to five subscales of Negative Emotions, Social Pressure, Availability, Physical Discomfort, and Positive Activities (e.g., "I can resist eating when I am watching TV") range from 0 (Not confident) to 9 (Very confident), and are summed. Internal consistencies ranged from .70 to .90 (Clark et al., 1991), and the predictive validity was acceptable in multiple studies (Warziski, Sereika, Styn, Music, \& Burke, 2008).

Self-regulation for exercise and self-regulation for eating were separately measured by modified versions of a scale by Saelens and colleagues (2000), where 10 items each are based on the content of the treatment (e.g., "I set physical activity goals" and "I say positive things to myself about eating well", respectively). Responses ranged from 1 (Never) to 5 (Often). The internal consistencies were .78 and .81 , and test-retest reliabilities over 2 weeks were .78 and .74 , respectively (Annesi \& Marti, 2011).

Tension and fatigue. The Profile of Mood States Short Form (McNair \& Heuchert, 2005) 5-item scales of Tension (e.g., anxious) and Fatigue (e.g., worn-out) required responses from 0 (Not at all) to 4 (Extremely) to indicate "how you have been feeling during the past week including today". Internal consistencies were .91 and .93 , and test-retest reliabilities over 3 weeks were .70 and .66 , respectively (McNair \& Heuchert, 2005). Concurrent validity was demonstrated with a variety of accepted instruments.

$B M I$ was calculated as weight in $\mathrm{kg} /$ height in $\mathrm{m}^{2}$. A recently calibrated digital scale was used to measure weight, and a stadiometer was used to measure height.

\section{Procedure}

Each participant was provided full access to a YMCA wellness facility and received an orientation to study processes associated with his/her assigned group. The exercise support component was identical in both the Nutrition Education and Behavioral Nutrition groups. It consisted of a previously tested protocol of cognitive-behavioral methods within six, 1-hour meetings with a trained YMCA wellness leader, spaced across 26 weeks (Coach Approach; Annesi \& Unruh, 2007). The one-on-one sessions included an orientation to exercise apparatus and setting an exercise plan. Widely used recommendations for volume of weekly exercise (150 minutes of moderate cardiovascular physical activity, Garber et al., 2011) were described, but it was clearly indicated that any volume of exercise may be beneficial - especially initially. Most time, however, was spent on an array of self-regulatory/self-management methods intended to promote adherence. Long-term exercise goals were identified, documented, and broken down into process-oriented short-term goals where ongoing progress was tracked graphically to increase feelings of competence. Instruction in additional self-regulatory skills such as cognitive restructuring, stimulus control, self-reward, and relapse prevention were given during the sessions. According to the taxonomy of behavior change techniques suggested by Abraham and Mi- 
chie (2008), the following techniques were applied: barrier identification, encouragement, behavioral goals, specific goal setting, establishing graded tasks, instruction in desired behaviors, self-monitoring behaviors, performance feedback, use of prompts or cues, behavioral contracting, social supports, self-talk, relapse prevention, stress management, and time management.

The nutrition component of the treatments varied by group. In the Nutrition Education group, a standardized program of six, 1-hour group sessions of nutrition education (Kaiser Permanente Health Education Services, 2008) was administered over 12 weeks. Sessions began 5 to 6 weeks after initiation of the exercise support component and were led by trained YMCA wellness leaders. Program components included understanding macronutrients, using the U.S. Food Guide Pyramid, and menu planning. An emphasis was placed on educating participants in healthy eating.

The Behavioral Nutrition group had the identical format and meeting times as the Nutrition Education group. Treatment components differed, however, and instead of education on healthy eating they primarily focused on cognitive-behavioral techniques such as setting caloric goals and logging daily food and calorie intake, regular self-weighing, cognitive restructuring, relapse prevention training, cues to overeating, and relaxation strategies. The array of eating behavior change techniques used was similar to that of the exercise support component.

Wellness leaders were trained in only one of the nutrition protocols along with the exercise support protocol, and were blind to the purposes of the investigation. Compliance with treatment protocols was monitored by YMCA supervisors in cooperation with study administrators. Psychological and physiological assessments were administered at baseline, Week 13, and Week 26.

\section{Data Analyses}

An intention-to-treat design was used. Consistent with related research (Nackers, Ross, \& Perri, 2010; Teixeira et al., 2010), the conservative last-observation-carried forward method for imputation, recently used in related research was incorporated. ${ }^{1}$ Based on recent suggestions (Glymor, Weuve, Berkman, Kawachi, \& Robins), change scores were calculated as the non-adjusted difference between scores at baseline (Time 1) and scores at Week 13 (for self-regulation for exercise and Exercise Self-Efficacy) or Week 26 (for self-regulation for eating, self-efficacy for controlled eating [Weight Efficacy Lifestyle], and BMI) (each designated as Time 2). These differing intervals were chosen because 1) treatments were designed to affect self-regulation and self-efficacy for exercise prior to self-regulation and self-efficacy for controlled eating, and 2) previous research (Oaten \& Cheng, 2006; Teixeira et al., 2010) and theory (Hagger et al., 2009) suggests a carry-over effect in the direction of self-regulation and self-efficacy for exercise to self-regulation and self-efficacy for eating. Statistical significance was set at $p<.050$, throughout. Because this research was probative, findings of $p=.051$ to .100 were also reported.

Initially, a series of mixed-model repeated measures ANOVAs was conducted to assess changes in the self-regulation and self-efficacy measures, and BMI, over time, and whether these changes differed by treatment condition. Regression analyses were then conducted to assess the relationship between changes

${ }^{1}$ It should be noted that results did not differ when multiple imputation, which has been considered preferable by some (Schafer \& Graham, 2002), was used for imputation of missing values. in self-regulation for exercise and self-regulation for eating, and exercise self-efficacy and self-efficacy for controlled eating; and whether those relationships were affected by treatment condition. In the primary analyses, Tension and Fatigue scores (mean scores from baseline, Week 13, and Week 26) were separately evaluated as moderators of the relationships of score changes in self-regulation for exercise with self-regulation for controlled eating, and exercise self-efficacy with self-efficacy for controlled eating. As suggested by Baron and Kenny (1986) and Frazier, Tix, and Barron (2004), in Step 1 of each of the corresponding multiple regression equations, the predictor and moderator were simultaneously entered. These variables were subsequently centered and standardized via $Z$-score transformation $\left(\mathrm{X}-M_{\mathrm{X}} / S D_{\mathrm{X}}\right)$ prior to being multiplied with one another to create an interaction term. This interaction term was then forced into the multiple regression equation in Step 2 to determine if it significantly added to the variance explained in Step 1 . Thus, if the associated change in $R^{2}$ was significant, then significant moderation was identified.

A multiple regression analysis was also conducted to determine the proportion of the variance in BMI change accounted for by changes in self-regulation for eating and self-efficacy for controlled eating.

\section{Results}

No significant group difference was found at baseline. There were significant over-time improvements in self-regulation for exercise, self-regulation for controlled eating, Exercise SelfEfficacy, and Weight Efficacy Lifestyle (self-efficacy for controlled eating) scores (all $p \mathrm{~s}<.001$ ), however none of the changes differed by treatment condition (see Table 1 for descriptive statistics). Using data aggregated across groups, there were strong relationships found between scores of changes in self-regulation for exercise and self-regulation for controlled eating ( $\beta$ $=.63, S E=.04, p<.001)$, and changes in Exercise Self-Efficacy and Weight Efficacy Lifestyle $(\beta=.51, S E=.19, p<.001)$. Group membership did not significantly affect these relationships.

All linear bivariate relationships between scores on the independent variables of Tension $(M=4.97, S D=4.50)$ and Fatigue $(M=12.32, S D=7.25)$, and the dependent variables of changes in Exercise Self-Efficacy and Weight Efficacy Lifestyle, were inverse $(r \mathrm{~s}=-.08$ to -.14$)$, indicating that as mood scores were less favorable, improvements in self-regulation and self-efficacy for controlled eating were less. Both Tension and Fatigue scores significantly moderated the relationship between changes in self-regulation for exercise and self-regulation for controlled eating (Table 2). Tension and Fatigue each approached significant moderation of the relationship between changes in Exercise Self-Efficacy and Weight Efficacy Lifestyle scores (Table 2).

Reduction in BMI was significant, $F(1,202)=89.50$, $p<.001$. The Behavioral Nutrition group had a greater reduction that was marginally significant, $F(1,202)=3.01, p=.084$. For all participants aggregated, both change in self-regulation for controlled eating $(\beta=-.35, S E=.02, p<.001)$ and Weight Efficacy Lifestyle $(\beta=-.26, S E=.004, p<.001)$ significantly contributed to the explained variance in BMI change $\left(R^{2}=.30\right.$, $p<.001)$.

\section{Discussion}

Consistent with previous research with adults across degrees 
Table 1.

Changes in self-regulation and self-efficacy measures.

\begin{tabular}{|c|c|c|c|c|c|c|c|}
\hline & \multirow[t]{2}{*}{ Possible score range } & \multicolumn{2}{|c|}{ Time 1} & \multicolumn{2}{|c|}{ Time 2} & \multicolumn{2}{|c|}{ Change score } \\
\hline & & $M$ & $S D$ & $M$ & $S D$ & $M_{\text {change }}$ & $S D$ \\
\hline \multicolumn{8}{|c|}{ Nutrition Education group $(n=95)$} \\
\hline Self-regulation for exercise & $10-50$ & 20.08 & 5.96 & 25.78 & 8.04 & 5.70 & 8.09 \\
\hline Self-regulation for eating & $10-50$ & 21.50 & 6.33 & 25.81 & 7.09 & 4.31 & 5.62 \\
\hline Exercise Self-Efficacy & $5-35$ & 28.12 & 11.38 & 31.54 & 11.49 & 3.42 & 10.17 \\
\hline Weight Efficacy Lifestyle & $0-180$ & 96.07 & 34.15 & 115.83 & 33.70 & 19.76 & 32.61 \\
\hline BMI $\left(\mathrm{kg} / \mathrm{m}^{2}\right)$ & & 45.81 & 4.78 & 44.74 & 4.96 & -1.07 & 1.59 \\
\hline \multicolumn{8}{|c|}{ Behavioral Nutrition group $(n=109)$} \\
\hline Self-regulation for exercise & $10-50$ & 21.51 & 4.89 & 26.55 & 7.41 & 5.04 & 7.16 \\
\hline Self-regulation for eating & $10-50$ & 21.46 & 6.15 & 26.69 & 7.83 & 5.23 & 6.09 \\
\hline Exercise Self-Efficacy & $5-35$ & 29.69 & 10.98 & 32.59 & 11.17 & 2.90 & 10.28 \\
\hline Weight Efficacy Lifestyle & $0-180$ & 97.72 & 34.41 & 117.38 & 38.00 & 19.66 & 31.78 \\
\hline $\operatorname{BMI}\left(\mathrm{kg} / \mathrm{m}^{2}\right)$ & & 46.24 & 5.02 & 44.68 & 5.30 & -1.56 & 2.27 \\
\hline
\end{tabular}

Table 2.

Moderation analyses of Tension and Fatigue on relationships of changes in exercise-related and eating-related self-regulation and self-efficacy $(\mathrm{N}=$ 204).

\begin{tabular}{|c|c|c|c|c|c|c|c|}
\hline & $\mathrm{B}$ & $S E \mathrm{~B}$ & $\beta$ & $R^{2}$ & $p$ & $\Delta R^{2}$ & $p$ \\
\hline \multicolumn{8}{|c|}{ Relationship of changes in self-regulation for exercise with self-regulation for controlled eating } \\
\hline \multicolumn{8}{|l|}{ Tension as moderator } \\
\hline Step 1 & & & & .39 & $<.001$ & & \\
\hline$\Delta$ Self-regulation for exercise & 3.68 & .33 & .63 & & $<.001$ & & \\
\hline Tension & -.02 & .33 & .00 & & .964 & & \\
\hline Step 2 & & & & .42 & $<.001$ & .03 & .002 \\
\hline$\Delta$ Self-regulation for exercise & 3.83 & .32 & .65 & & $<.001$ & & \\
\hline Tension & .23 & .33 & .04 & & .491 & & \\
\hline$\Delta$ Self-regulation for exercise $\times$ Tension & 1.10 & .35 & .17 & & .002 & & \\
\hline \multicolumn{8}{|l|}{ Fatigue as moderator } \\
\hline Step 1 & & & & .39 & $<.001$ & & \\
\hline$\Delta$ Self-regulation for exercise & 3.64 & .33 & .62 & & $<.001$ & & \\
\hline Fatigue & -.27 & .33 & -.05 & & .401 & & \\
\hline Step 2 & & & & .41 & $<.001$ & .02 & .010 \\
\hline$\Delta$ Self-regulation for exercise & 3.78 & .33 & .64 & & $<.001$ & & \\
\hline Fatigue & .10 & .35 & .02 & & .768 & & \\
\hline$\Delta$ Self-regulation for exercise $\times$ Fatigue & 1.09 & .42 & .16 & & .010 & & \\
\hline \multicolumn{8}{|c|}{ Relationship of changes in Exercise Self-Efficacy with Weight Efficacy Lifestyle scores } \\
\hline \multicolumn{8}{|l|}{ Tension as moderator } \\
\hline Step 1 & & & & .26 & $<.001$ & & \\
\hline$\Delta$ Exercise Self-Efficacy & 16.13 & 1.97 & .50 & & $<.001$ & & \\
\hline Tension & -1.51 & 1.97 & -.05 & & .442 & & \\
\hline Step 2 & & & & .28 & $<.001$ & .02 & .053 \\
\hline$\Delta$ Exercise Self-Efficacy & 17.12 & 2.02 & .53 & & $<.001$ & & \\
\hline Tension & -.98 & 1.97 & -.03 & & 621 & & \\
\hline$\Delta$ Exercise Self-Efficacy $\times$ Tension & 4.57 & 2.35 & .12 & & .053 & & \\
\hline \multicolumn{8}{|l|}{ Fatigue as moderator } \\
\hline Step 1 & & & & .26 & $<.001$ & & \\
\hline$\Delta$ Exercise Self-Efficacy & 16.42 & 1.98 & .51 & & $<.001$ & & \\
\hline Fatigue & .36 & 1.98 & .01 & & .857 & & \\
\hline Step 2 & & & & .27 & $<.001$ & .01 & .071 \\
\hline$\Delta$ Exercise Self-Efficacy & 17.24 & 2.02 & .54 & & $<.001$ & & \\
\hline Fatigue & .83 & 1.98 & .03 & & 677 & & \\
\hline$\Delta$ Exercise Self-Efficacy $\times$ Fatigue & 4.20 & 2.32 & .11 & & .071 & & \\
\hline
\end{tabular}


of overweight (Klem, Wing, McGuire, Seagle, \& Hill, 1997), the use of exercise for improving behavioral predictors of reduced weight in adults with morbid obesity was supported. More specifically, findings indicated that cognitive-behavioral exercise support was associated with significant improvements in self-regulation for exercise, exercise self-efficacy, selfregulation for eating, and self-efficacy for controlled eating. One explanation for the lack of a significantly greater increase in self-efficacy and self-regulation for controlled eating associated with the cognitive-behaviorally based nutritional methods vs. simply nutrition education is a ceiling effect. Possibly the supported exercise induced improvements in self-regulation and self-efficacy for controlled eating to a point that is difficult to further increase. This, however, requires direct investigation. As expected, the relations between changes in self-regulation for exercise and self-regulation for controlled eating, and changes in exercise self-efficacy and self-efficacy for controlled eating, were strong. Whether specific types of self-efficacy (e.g., to resist social pressure to overeat) or self-regulatory skills (e.g., productive self-talk) have especially strong carryover effects from exercise to eating may also be an important topic for further research, and may help to refine the foci of the treatment. When taxonomies of behavior change methods (e.g., Abraham \& Michie, 2008) are better reflected in validated inventories, effects emanating from corresponding treatment components may be analyzed and used to evolve methods to improve outcomes. For example, Annesi (2011) suggested that goal setting methods defined as "review of behavioral goals" and "feedback on [goal-related] performance" (Abraham \& Michie, 2008) are associated with increased self-efficacy. Presently, even "state-of-the-art" cognitive-behavioral treatments for weight loss (e.g., Cooper, Fairburn, \& Hawker, 2003) incorporate a quite generic array of techniques that, even over decades of research, generally fail in their goal of sustained weight loss (Cooper et al., 2010).

Because higher levels of tension and fatigue demonstrated tendencies for diminishing the newly identified relationships between exercise- and eating-related self-regulation and selfefficacy in persons with Grade 3 obesity, assessment of these mood factors prior to, and throughout, intervention may be warranted for this subgroup. Hopefully, appropriate methods (e.g., deep breathing for heightened tension; energizing imagery for heightened fatigue) may judiciously be applied so that carry-over effects from exercise to controlled eating may be maximized. Continued research in these areas may also eventually uncover reasons why exercise is the strongest predictor of sustained weight loss. It has been suggested that negotiating maintained exercise may nurture self-efficacy and self-regulatory skills prior to their being required for overcoming adverse eating behaviors related to a disappointing plateau in weightloss shortly after treatment initiation (Annesi \& Marti, 2011). The use of research to simultaneously advance theory and treatment in this manner has been specifically suggested (Baranowski, Lin, Wetter, Resnicow, \& Hearn, 1997).

Limitations of the research were, however, present. Although also interpreted as a strength (Glasgow, 2008), the use of a field setting challenged internal validity. For example, social support and expectation effects by both instructors and fellow participants were not controllable, thus their impact on findings is unknown. Also, participants were volunteers, which may have been associated with high motivation and/or other personal characteristics that may have influenced results. Although difficult, the use of a true control condition may mitigate these limitations in extensions of this research. It will also be impor- tant to determine if findings generalize to individuals with disorders such as diabetes and cardiorespiratory disease, in addition to Grade 3 obesity. Extensions of this research will also be required to determine the minimum dosages of exercise and professionally led intervention required for significant effects. Because adherence to exercise is problematic (Annesi \& Unruh, 2007), and may be particularly difficult for persons with morbid obesity, minimum volumes of physical activity for acceptable effects should be carefully considered (rather than standard guidelines [i.e., Garber et al., 2011] be relied upon). This is particularly important as only maintained exercise is a correlate of sustained weight loss (Klem et al., 1997). Although addressed in samples of a lower weight (Annesi \& Marti, 2011), the directionality if psychosocial changes from exercise- to eating-related should be addressed beyond the theoretically based assumptions of temporality made within the measurement of self-regulation and self-efficacy within this research.

Due to the clear failure of traditional behavioral methods (Cooper et al., 2010; Mann et al., 2007), the invasive and expensive procedure of bariatric surgery is now often the "treatment-of-choice" for adults with Grade 3 obesity (Maggard et al., 2005). Thus, it is the responsibility of behavioral science to better understand the mechanisms for success with behavioral treatments, and translate these findings into more effective, efficient, and reliable interventions for that population. The present findings have the prospect of contributing to this important task. As suggested by theory (Baker \& Brownell, 2000), review of previous research (Mann et al., 2007), and the present findings, it is possible that cognitively supported exercise may be leveraged for weight loss in ways not previously identified.

\section{References}

Abraham, C., \& Michie, S. (2008). A taxonomy of behavior change techniques used in interventions. Health Psychology, 27, 379-387. doi:10.1037/0278-6133.27.3.379

American College of Sports Medicine (2009). Appropriate physical activity intervention strategies for weight loss and prevention of weight regain for adults. Medicine \& Science in Sports \& Exercise, 42, 459-471

Annesi, J. J. (2011). Self-regulatory skills usage strengthens the relations of self-efficacy for improved eating, exercise, and weight in the severely obese: Toward an explanatory model. Behavioral Medicine, 37, 71-76. doi: $10.1080 / 08964289.2011 .579643$

Annesi, J. J., \& Marti, C. N. (2011). Path analysis of exercise treatment-induced changes in psychological factors leading to weight loss. Psychology and Health, 26, 1081-1098.

doi: $10.1080 / 08870446.2010 .534167$

Annesi, J. J., \& Unruh, J. L. (2007). Effects of The Coach Approach intervention on drop out rates among adults initiating exercise programs at nine YMCAs over three years. Perceptual and Motor Skills, 104, 459-466. doi:10.2466/pms.104.2.459-466

Annesi, J. J., \& Whitaker, A. C. (2010). Psychological factors discriminating between successful and unsuccessful weight loss in a behavioral exercise and nutrition education treatment. International Journal of Behavioral Medicine, 17, 168-175. doi:10.1007/s12529-009-9056-2

Baker, C. W., \& Brownell, K. D. (2000). Physical activity and maintenance of weight loss: Physiological and psychological mechanisms. In C. Bouchard (Ed.), Physical activity and obesity (pp. 311-328). Champaign, IL: Human Kinetics.

Bandura, A. (1986). Social foundations of thought and action: A social cognitive theory. Englewood Cliffs, NJ: Prentice Hall.

Bandura, A. (1997). Self-efficacy: The exercise of control. New York, NY: Freeman.

Baranowski, T., Lin, L. S., Wetter, D. W., Resnicow, K., \& Hearn, M. 
D. (1997). Theory as mediating variables: Why aren't community interventions working as desired? Annals of Epidemiology, 7, S89S95. doi:10.1016/S1047-2797(97)80011-7

Baron, R. M., \& Kenny, D. A. (1986). The moderator-mediator variable distinction in social psychological research: Conceptual, strategic, and statistical considerations. Journal of Personality and Social Psychology, 51, 1173-1182. doi:10.1037/0022-3514.51.6.1173

Bult, M. J., van Dalen, T., \& Muller, A. F. (2008). Surgical treatment of obesity. European Journal of Endocrinology, 158, 135-145. doi:10.1530/EJE-07-0145

Clark, M. M., Abrams, D. B., Niaura, R. S., Eaton, C. A., \& Rossi, J. S. (1991). Self-efficacy in weight management. Journal of Consulting and Clinical Psychology, 59, 739-744. doi:10.1037/0022-006X.59.5.739

Cooper, Z., Doll, H. A., Hawker, D. M., Byrne, S., Bonner, G., Eeley, E., Fairburn, C. G. et al. (2010). Testing a new cognitive behavioural treatment for obesity: A randomized controlled trial with three-year follow-up. Behavior Research and Therapy, 48, 706-713. doi:10.1016/j.brat.2010.03.008

Cooper Z., Fairburn C. G., \& Hawker, D. M. (2003). Cognitive-behavioral treatment of obesity: A clinician's guide. New York: Guilford.

Davies, M. (2007). Psychological assessment of candidates for bariatric surgery. Irish Journal of Psychological Medicine, 24, 99-102.

Flegal, K. M., Carroll, M. D., Ogden, C. L., \& Curtin, L. R. (2010). Prevalence and trends in obesity among US adults, 1999-2008. Journal of the American Medical Association, 303, 235-241. doi:10.1001/jama.2009.2014

Fogelholm, M., \& Kukkomen-Harjula, K. (2000). Does physical activity prevent weight gain-A systematic review. Obesity Reviews, 1, 95-111. doi:10.1046/j.1467-789x.2000.00016.x

Frazier, P. A., Tix, A. P., \& Barron, A. P. (2004). Testing moderator and mediator effects in counseling psychological research. Journal of Counseling Psychology, 51, 115-134. doi:10.1037/0022-0167.51.1.115

Garber, C. E., Blissmer, B., Deschenes, M. R. Franklin, B. A., Lamonte, Michael, J., David P. et al. (2011). Quantity and quality of exercise for developing and maintaining cardiorespiratory, musculoskeletal, and neuromotor fitness in apparently healthy adults: Guidance for prescribing exercise. Medicine \& Science in Sports \& Exercise, 43, 1334-1359. doi:10.1249/MSS.0b013e318213fefb

Glasgow, R. E. (2008). What types of evidence are most needed to advance behavioral medicine? Annals of Behavioral Medicine, 35, $19-25$.

Glasgow, R. E., \& Emmons, K. M. (2007). How can we increase translation of research into practice? Types of evidence needed. Annual Review of Public Health, 28, 413-433. doi:10.1146/annurev.publhealth.28.021406.144145

Glymor, M. M., Weuve, J., Berkman, L. F., Kawachi, I., \& Robins, J. M. (2005). When is baseline adjustment useful in analyses of change? An example with education and cognitive change. American Journal of Epidemiology, 162, 267-278. doi:10.1093/aje/kwi187

Greenberg, I., Perna, F., Kaplan, M., \& Sullivan, M. A. (2005). Behavioral and psychological factors in the assessment and treatment of obesity surgery patients. Obesity Research, 13, 244-249. doi:10.1038/oby.2005.33

Hagger, M. S., Wood, C., Stiff, C., \& Chatzisarantis, N. L. D. (2010). Ego depletion and the strength model of self-control: A meta-analysis. Psychological Bulletin, 136, 495-525. doi:10.1037/a0019486

Hankonen, N., Absetz, P., Haukkala, A., \& Uutela, A. (2009). Socioeconomic status and psychosocial mechanisms of lifestyle change in a Type 2 diabetes prevention trial. Annals of Behavioral Medicine, 38, 160-165. doi:10.1007/s12160-009-9144-1
Kaiser Permanente Health Education Services (2008). Cultivating Health weight management kit (8th ed.). Portland, OR: Kaiser Permanente.

Klem, M. L., Wing, R. R., McGuire, M. T., Seagle, H. M., \& Hill, J. O. (1997). A descriptive study of individuals successful at long-term maintenance of substantial weight loss. American Journal of Clinical Nutrition, 66, 239-246.

Maggard, M. A., Shurgarman, L. R., Suttorp, M., Maglione, A., Sugerman, H. H., Livingston, E. J., Li, Z. et al. (2005). Meta-analysis: Surgical treatment of obesity. Annals of Internal Medicine, 142, 547559.

Mann, T., Tomiyama, J., Westling, E., Lew, A. M., Samuels, B., \& Chatman, J. (2007). Medicare's search for effective obesity treatments: Diets are not the answer. American Psychologist, 62, 220-233. doi:10.1037/0003-066X.62.3.220

Marcus, B. H., Selby, V. C., Niaura, R. S., \& Rossi, J. S. (1992). Selfefficacy and the stages of exercise behavior change. Research Quarterly for Exercise and Sport, 63, 60-66.

McAuley, E., \& Mihalko, S. L. (1998). Measuring exercise-related self-efficacy. In J. L. Duda (Ed.), Advances in sport and exercise psychology measurement (pp. 371-390). Morgantown, WV: Fitness Information Technology.

McNair, D. M., \& Heuchert, J. W. P. (2005). Profile of Mood States technical update. North Tonawanda, NY: Multi-Health Systems.

Mokdad, A. H., Ford, E. S., Bowman, B. A., Dietz, W. H., Vinicor, F., Bales, V. S., \& Marks, J. S. (2003). Prevalence of obesity, diabetes, and obesity-related health risk factors, 2001. Journal of the American Medical Association, 289, 76-79. doi:10.1001/jama.289.1.76

Nackers, L. M., Ross, K. M., \& Perri, M. G. (2010). The impact of rate of initial weightloss on long-term success in obesity treatment: Does slow and steady win the race? International Journal of Behavioral Medicine, 17, 161-167. doi:10.1007/s12529-010-9092-y

Oaten, M., \& Cheng, K. (2006). Longitudinal gains in self-regulation from regular physical exercise. British Journal of Health Psychology, 11, 717-733. doi:10.1348/135910706X96481

Puhl, R., \& Brownell, K. D. (2002). Stigma, discrimination, and obesity. In C. G. Fairburn, \& K. D. Brownell (Eds.). Eating disorders and obesity (2nd ed., pp. 108-112). New York: Guilford.

Saelens, B. E., Gehrman, C. A., Sallis, J. F., Calfas, K. J., Sarkin, J. A., $\&$ Caparosa, S. (2000). Use of self-management strategies in a 2-year cognitive-behavioral intervention to promote physical activity. Behavior Therapy, 31, 365-379. doi:10.1016/S0005-7894(00)80020-9

Schafer, J. L., \& Graham, J. W. (2002). Missing data: Our view of the state of the art. Psychological Methods, 7, 147-177. doi:10.1037/1082-989X.7.2.147

Svetkey, L. P., Stevens, V. J., Brantley, P. J., Appel, L. J., Hollis, J. F., Loria, C. M., Aicher, K. et al. (2008). Comparison of strategies for sustaining weight loss. Journal of the American Medical Association, 299, 1139-1148. doi:10.1001/jama.299.10.1139

Teixeira, P. J., Silva, M. N., Coutinho, S. R., Palmeira, A. L., Mata, J., Viera, P. N., Sardinha, L. B. et al. (2010). Mediators of weight loss and weight loss maintenance in middle-aged women. Obesity, 18, 725-735. doi: $10.1038 /$ oby.2009.281

Tice, D. M., \& Bratslavsky, E. (2000). Giving in to feel good: The place of emotion regulation in the context of general self-control. Psychological Inquiry, 11, 149-159. doi:10.1207/S15327965PLI1103 03

Warziski, M. T., Sereika, S. M., Styn, M. A., Music, E., \& Burke, L. E. (2008). Changes in self-efficacy and dietary adherence: The impact on weight loss in the PREFER study. Journal of Behavioral Medicine, 31, 81-92. doi:10.1007/s10865-007-9135-2 